Neusa Aparecida de Sousa Basso ${ }^{1}$

Roberto Antonio AraúJo Costa ${ }^{2}$

Cláudia Garcia Magalhães ${ }^{3}$

Marilza Vieira Cunha RudGe ${ }^{4}$

Iracema de Mattos Paranhos Calderon ${ }^{5}$

Artigos originais

Palavras-chaves

Insulina/uso terapêutico Insulina/administração \& dosagem

Diabetes mellitus

Diabetes gestacional

Gravidez

Glicemia/análise

Prognóstico

Keywords

Insulin/therapeutic use Insulin/administration \& dosage

Diabetes mellitus

Diabetes, gestational

Pregnancy

Blood glucose/analysis

Prognosis

Neusa Aparecida de Sousa Basso Rua Dona Virgínia Ferraz de Almeida Prado, 161 Jardim São Francisco

(EP 17209-290 - Jaú/SP

Fone: (14) 3621-7321

E-mail: neusousabasso@bol.com.br

Recebido

08/01/2007

Aceito com modificações

28/05/2007

\section{Insulinoterapia, controle glicêmico materno e prognóstico perinatal - diferença entre o diabetes gestacional e o clínico}

\author{
Insulinotherapy, maternal glycemic control and perinatal prognosis \\ - difference between clinical and gestational diabetes
}

\section{Resumo}

OBJETIVO: avaliar protocolo de insulinoterapia e conseqüentes resultados maternos e perinatais, no diabetes gestacional e clínico, num serviço de referência para gravidez de alto risco. MÉTODOS: estudo prospectivo e descritivo, incluindo 103 gestantes portadoras de diabetes gestacional ou clínico, tratadas com insulina e acompanhadas no serviço, no período de outubro de 2003 a dezembro de 2005. Foram excluídos casos de gemelaridade, abortamento, abandono do pré-natal e parto fora do serviço. Compararam-se idade gestacional no início do tratamento; dose, aplicações/dia e incremento de insulina $(U \mathrm{U} / \mathrm{kg})$; média glicêmica e resultados perinatais. Utilizaram-se ANOVA e testes de Fisher e Goodman, considerando p<0,05. RESULTADOS: a multiparidade (92 versus 67,9\%), o índice de massa corporal (IMC) pré-gestacional $>25 \mathrm{~kg} / \mathrm{m}^{2}$ (88 versus $58,5 \%$ ), o ganho de peso $<8 \mathrm{~kg}$ (36 versus $17 \%$ ) e o maior incremento de insulina caracterizaram o diabetes gestacional. No diabetes clínico, apesar da maior proporção de média glicêmica $\geq 120 \mathrm{mg} / \mathrm{dL}(39,2$ versus 24\%) no final da gestação, a insulinoterapia teve início precoce (47,2 versus 4\%), maior duração (56,6 versus 6\%), e maior dose diária de insulina (92 versus 43 UI/dia), administrada em até três aplicações/ dia $(54,7$ versus 16,0). A macrossomia foi maior entre os recém-nascidos do grupo diabetes gestacional ( 16 versus $3,8 \%$, sendo o único resultado neonatal significativo. Não houve óbito neonatal e o único caso de óbito fetal ocorreu no diabetes clínico. As demais complicações neonatais não diferenciaram os grupos e a maioria dos recém-nascidos recebeu alta em até sete dias (46\% versus 55,8\%). CONCLUSÕES: a análise desta série de casos identificou diferenças no protocolo de insulinoterapia em relação à quantidade (UI/dia), à dose (UI/kg de peso) e ao número de aplicações diárias, mais acentuadas no diabetes clínico, e ao incremento de insulina, maior no diabetes gestacional. De modo indireto, a qualidade do controle glicêmico materno e os resultados perinatais satisfatórios atestaram que o protocolo de tratamento foi adequado e não dependeu do tipo de diabetes.

\section{Abstract}

PURPOSE: to evaluate the insulin therapy protocol and its maternal and perinatal outcome in patients with clinical or gestational diabetes in a high risk reference service. METHODS: descriptive and prospective study including 103 pregnant women with gestational or clinical diabetes treated with insulin and attended by the reference service from October 2003 to December 2005. Gemellarity, miscarriages, unfinished prenatal care and deliveries not attended by the service were excluded. The gestational age at the beginning of the treatment, dosage, doses/day, increment of insulin (UI/kg), glycemic index (GI) and perinatal outcomes were compared. ANOVA, Fisher's exact test and Goodman's test considering $\mathrm{p}<0.05$ were used. RESULTS: multiparity (92 versus $67.9 \%$ ), pre-gestational body mass index (BMI) $>25 \mathrm{~kg} / \mathrm{m}^{2}$ (88 versus 58.5\%), weight gain (WG) <8 kg (36 versus 17\%) and a high increment of insulin characterized the gestational diabetes. For the patients with clinical diabetes, despite the highest Gl (120 mg/dL (39.2 versus 24\%)) at the end of the gestational period, insulin therapy started earlier (47.2 versus $4 \%$ ), lasted longer (56.6 versus $6 \%$ ) and higher doses of insulin (92 versus $43 \mathrm{UI}$ /day) were administered up to three times a day (54.7 versus 16\%). Macrosomia was higher among newborns from the cohort of patients with gestational diabetes (16 versus 3.8\%), being the only significant neonatal outcome. There were no neonatal deaths, except for one fetal death in the cohort of patients with

Mestre em Obstetrícia pelo Programa de Pós-Graduação em Ginecologia, Obstetrícia e Mastologia da Faculdade de Medicina de Botucatu da Universidade Estadual Paulista "Júlio Mesquita Filho" - UNESP - Botucatu (SP), Brasi

2Professor Assistente do Serviço de Diabete e Gravidez, Disciplina de Obstetrícia, do Departamento de Ginecologia e Obstetrícia da Faculdade de Medicina de Botucatu da Universidade Estadual Paulista "Júlio Mesquita Filho" - UNESP - Bołucatu (SP), Brasil.

${ }_{3}^{3}$ Médico Obstetra do Serviço de Diabete e Gravidez do Departamento de Ginecologia, Obstetrícia e Mastologia da Faculdade de Medicina de Botucatu da Universidade Estadual Paulista "Júlio Mesquita Filho" - UNESP - Botucatu (SP), Brasil.

4Professora Titular do Serviço de Diabete e Gravidez, Disciplina de Obstetrícia, do Departamento de Ginecologia e Obstetrícia da Faculdade de Medicina de Botucatu da Universidade Estadual Paulista "Júlio Mesquita Filho" - UNESP - Botucatu (SP), Brasil.

SProfessora Livre-Docente, do Serviço de Diabete e Gravidez, do Departamento de Ginecologia e Obstetrícia da Faculdade de Medicina de Botucatu da Universidade Estadual Paulista "Júlio Mesquita Filho" - UNESP - Botucału (SP), Brasil. 
clinical diabetes. There were no differences in the other neonatal complications in both cohorts, and most of the newborns were discharged from hospital up to seven days after delivery (46\% versus 55.8\%). CONCLUSIONS: the analysis of these two cohorts has shown differences in the insulin therapy protocol in quantity (UI/day), dosage (UI/ kg weight) and number of doses/day, higher for the clinical diabetes cohort, and in the increment of insulin, higher for the gestational diabetes cohort. Indirectly, the quality of maternal glycemic control and the satisfactory perinatal outcome have proven that the treatment protocol was adequate and did not depend on the type of diabetes.

\section{Introdução}

Diabetes mellitus é uma doença sistêmica que envolve alterações no metabolismo de carboidratos, lipídios, proteínas e eletrólitos. De caráter crônico e evolutivo, caracteriza-se por deficiência na secreção e/ou ação da insulina, com conseqüente hiperglicemia. É um processo patológico que, durante a gestação, pode repercutir na saúde materna, fetal e perinatal ${ }^{1}$.

A gestação é um estado de hiperfunção pancreática, caracterizado por aumento da resistência periférica à insulina, parcialmente explicada pela presença dos hormônios diabetogênicos, entre eles: progesterona, cortisol, prolactina e lactogênio placentário. $\mathrm{Na}$ gestação, os níveis glicêmicos de jejum tendem a ser mais baixos e os valores pós-prandiais são elevados, havendo necessidade de maior produção/liberação de insulina. Nas gestantes em que não há incremento adequado na produção/liberação de insulina, diagnostica-se o diabetes mellitus gestacional (DMG), definido como qualquer grau de intolerância à glicose com início ou primeiro reconhecimento na gravidez ${ }^{1}$. Essa definição independe da idade gestacional e do tratamento utilizado para o controle da glicemia materna, não excluindo, portanto, a possibilidade de uma intolerância à glicose não-diagnosticada ser classificada como DMG. Para definir o diagnóstico, recomenda-se a reclassificação da paciente após seis semanas do parto e, na maioria dos casos, a normoglicemia será a regra ${ }^{2,3}$.

Quando associados à gestação, tanto o diabetes mellitus prévio (tipo 1 ou 2) como o DMG resultam em comprometimento materno e fetal. A hiperglicemia materna leva à hiperglicemia e conseqüente hiperinsulinemia fetal. A associação favorece o crescimento fetal exagerado e, entre outras complicações, a macrossomia, o aumento das taxas de cesárea, os traumas de canal de parto e a ocorrência de resultados neonatais adversos. Entre os mais comuns, destacam-se: hipoglicemia, hiperbilirrubinemia, hipocalcemia, policitemia e distúrbios respiratórios. As malformações congênitas dependem da presença de hiperglicemia materna no início da gestação e da qualidade de seu controle, sendo mais comuns no diabetes prévio à gestação. Além destas complicações, destaca-se o maior risco de óbito fetal e neonatal nas gestações associadas ao diabetes mal controlado ${ }^{2-4}$.

A literatura apresenta vários critérios para a qualidade do controle da glicemia materna no diabetes. A normoglicemia é definida por limites de $90 \mathrm{mg} / \mathrm{dL}$ na glicemia de jejum e de $130 \mathrm{mg} / \mathrm{dL}$ nas pós-prandiais de uma ou duas horas, avaliadas no perfil glicêmico (PG) diário ${ }^{5,6}$. Níveis glicêmicos de $95 \mathrm{mg} / \mathrm{dL}$ no jejum, $100 \mathrm{mg} / \mathrm{dL}$ nas pré-prandiais e 140 e $120 \mathrm{mg} /$ $\mathrm{dL}$, respectivamente, para as pós-prandiais de uma e duas horas são outros limites propostos ${ }^{2,3,7}$. A American Diabetes Association ${ }^{2}$ recomenda os valores de $105 \mathrm{mg} / \mathrm{dL}$ para o jejum, $155 \mathrm{mg} / \mathrm{dL}$ na pós-prandial de uma hora e $130 \mathrm{mg} / \mathrm{dL}$ na glicemia de duas horas após alimentação.

Os valores da média glicêmica (MG) também podem ser utilizados como indicadores na introdução ou ajuste da dose de insulina, permitindo avaliar a qualidade do controle glicêmico materno. Esse parâmetro é obtido pela média aritmética das glicemias dos PG de 24 horas (MG diária) ou de todas as glicemias avaliadas nos PG do trimestre (MG trimestral) ou da gestação (MG da gestação). Os limites de MG entre 100 e $120 \mathrm{mg} / \mathrm{dL}$ definem o controle adequado; os superiores a $120 \mathrm{mg} / \mathrm{dL}$, o controle inadequado, e os inferiores a $100 \mathrm{mg} / \mathrm{dL}$, o controle glicêmico ideal, diretamente relacionado ao prognóstico perinatal ${ }^{5,6,8}$.

A insulinoterapia está indicada nas gestantes portadoras de diabetes tipo 1 e, via de regra, substitui o anti-hiperglicemiante oral nas diabéticas do tipo 2, sempre em associação com dieta e exercício. No diabetes gestacional preconiza-se o uso de insulina humana, sempre que houver falha do controle glicêmico materno com a associação de dieta e atividade física. Entretanto, não há consenso nos protocolos, que orientam diferentes doses para cada trimestre e divergem no emprego do peso real ${ }^{9}$ ou do peso ideal ${ }^{10}$ para o cálculo da dose diária de insulina.

Considerando as controvérsias da literatura, é objetivo deste trabalho avaliar aspectos relacionados à insulinoterapia, ao controle glicêmico materno e aos conseqüentes resultados perinatais em gestantes portadoras de diabetes gestacional e clínico, acompanhadas em um Serviço de Diabete e Gravidez, referência terciária para estas gestações de risco. 


\section{Métodos}

Estudo prospectivo e descritivo, no qual se compararam aspectos relacionados ao protocolo de insulinoterapia de 103 gestantes portadoras de diabetes gestacional (classe A/B) ou clínico (classes B a FRH de Priscila White) atendidas no Serviço de Diabete e Gravidez da Faculdade de Medicina de Botucatu da Universidade Estadual Paulista (UNESP), no período de outubro de 2003 a dezembro de 2005.

O tamanho amostral foi calculado considerando-se a diferença de desvio padrão das doses iniciais e finais de insulina, observada em estudo anterior ${ }^{5}(18,7$ UI para o diabetes gestacional e 47,6 UI para o clínico); a diferença desejada entre as médias dos grupos, definida pela metade da diferença do desvio-padrão das doses iniciais e finais de insulina; o nível de significância de $10 \%$ e o poder estatístico de $80 \%$. Com esses dados, o tamanho amostral mínimo calculado foi de 50 gestantes para cada grupo. Foram incluídas 50 gestantes portadoras de diabetes gestacional e outras 53 com diabetes clínico (22 tipo 1 e 31 tipo 2), classificadas de acordo com as recomendações da American Diabetes Association ${ }^{1,2}$.

Para a seleção das pacientes, adotou-se o uso de insulina como critério único de inclusão. Foram excluídas da análise dos resultados as gestantes que não completaram o pré-natal e/ou não tiveram parto no serviço (abandono de tratamento), as gestações gemelares e os abortamentos. Para a comparação entre os dois grupos foram definidas variáveis maternas e perinatais, considerando-se características próprias das gestantes, aspectos da terapêutica insulínica, a qualidade do controle glicêmico materno e as conseqüentes repercussões perinatais.

Os dados maternos avaliados foram a idade ( 25 e $>25$ anos), a paridade (primípara ou multípara em relação à gestação avaliada), o índice de massa corporal (IMC) pré-gestacional ( 25 e $>25 \mathrm{~kg} / \mathrm{m}^{2}$ ) e o ganho de peso (GP) na gestação (adequado: 8 a $16 \mathrm{~kg}$ e inadequado: $<8 \mathrm{~kg}$ ou $>16 \mathrm{~kg})^{11}$.

A análise da terapêutica insulínica foi realizada considerando-se: a idade gestacional de início, diferenciada em até 12 , de 13 a 26 e mais de 27 semanas; a duração do tratamento (até 10 , de 11 a 20 e $\geq 21$ semanas); o número de aplicações diárias (uma, duas ou três) e a evolução do incremento de insulina na gestação, avaliada por curvas específicas. O comportamento fisiológico do incremento de insulina foi caracterizado por ascensão da curva de necessidade de insulina entre a 24 e a $36^{\mathrm{a}}$ semana de gestação; o não-fisiológico, por platô ou oscilação da curva entre a 24 e a $36^{a}$ semana de gestação ${ }^{12}$. Foram também analisados a dose total diária de insulina (UI/dia), as doses de insulina relacionadas ao peso atual/ real da gestante e o incremento de insulina (UI/ $\mathrm{kg}$ peso real), diferenciados para o primeiro, segundo e terceiro trimestres da gestação. Além disso, compararam-se as doses para base de cálculo de 0,5 (primeiro trimestre), 0,6 (segundo trimestre) e $0,7 \mathrm{UI} / \mathrm{kg}$ de peso real/dia (terceiro trimestre) do protocolo de insulinoterapia do Serviço, com as doses de 0,7 (primeiro trimestre), 0,8 (segundo trimestre) e $0,9 \mathrm{UI} / \mathrm{kg}$ de peso real/dia (terceiro trimestre), preconizadas em outro protocolo da literatura ${ }^{9}$.

A qualidade do controle glicêmico materno foi avaliada pela MG, calculada pela média aritmética de todas as glicemias dos PG - valor total das glicemias plasmáticas/número de dosagens glicêmicas, em cada trimestre gestacional. A MG foi classificada em ideal $(\mathrm{MG} \leq 100 \mathrm{mg} / \mathrm{dL})$, adequada $(100<\mathrm{MG}<120 \mathrm{mg} / \mathrm{dL})$ e inadequada $(\mathrm{MG} \geq 120 \mathrm{mg} / \mathrm{dL})^{5,6}$.

Entre os resultados perinatais, foram avaliados: a idade gestacional no nascimento; o peso do recém-nascido e sua classificação em relação à idade gestacional, categorizada em pequeno (peso $<\mathrm{P} 10$ ), adequado e grande (GIG; peso>P90); e a ocorrência de macrossomia (peso $\geq 4,0 \mathrm{~kg}$ ), hiperbilirrubinemia (presença de icterícia visível nas primeiras 24 horas de vida com bilirrubina plasmática maior que $12 \mathrm{mg} / \mathrm{dL}$ ), hipoglicemia (definida por uma única dosagem de glicemia igual ou inferior a $45 \mathrm{mg} / \mathrm{dL}$ nas primeiras 72 horas de vida) e policitemia (>60\%). A síndrome do desconforto respiratório (SDR) foi caracterizada por insuficiência respiratória de início precoce, imediatamente após o parto ou nas primeiras horas de vida com padrão radiológico típico, malformações e óbito (fetal e neonatal), além do tempo (dias) de internação do recém-nascido ${ }^{5,6,13}$.

A evolução da gestação (resultados maternos) foi anotada em protocolos específicos, preenchido a cada internação, e os resultados perinatais foram colhidos no puerpério, recorrendo-se ao livro de parto e aos prontuários dos recém-nascidos. Constituiu-se banco de dados, devidamente conferido e trabalhado para análise estatística no pacote estatístico SPSS. As médias foram comparadas por análise de variância (ANOVA), seguida pelo teste F; as proporções, pelo teste exato de Fischer (comparações 2x2) ou de Goodman (comparações múltiplas), adotando-se 5\% como limite de significância estatística $(\mathrm{p}<0,05)$.

Este projeto foi aprovado pelo Comitê de Ética em Pesquisa da Faculdade de Medicina de Botucatu da UNESP; todas as gestantes incluídas foram prévia e devidamente esclarecidas e assinaram o Termo de Consentimento Livre e Esclarecido.

A média da idade das gestantes do grupo diabetes gestacional foi de $32 \pm 3,8$ anos e do diabetes clínico, $29,4 \pm 6,5$ anos, e, independentemente do grupo, maior 
proporção delas (88 e 77,4\%) tinha idade superior a 25 anos. No diabetes gestacional, a maioria das gestantes era multípara (92\%) e apresentou IMC pré-gravídico superior a $25 \mathrm{~kg} / \mathrm{m}^{2}$ (88\%). Essas proporções foram significativamente diferentes das observadas no diabetes clínico, respectivamente, 67,9 e 58,5\%.

\section{Resultados}

Estes resultados referem-se à análise de 103 gestantes portadoras de diabetes, gestacional $(\mathrm{n}=50$; classes $\mathrm{A} / \mathrm{B})$ ou clínico ( $n=53$; classes B a FRH), tratadas com insulina e acompanhadas no Serviço de Diabete e Gravidez da Faculdade de Medicina de Botucatu da UNESP, no período de outubro de 2003 a dezembro de 2005.

O GP foi adequado para $42 \%$ das gestantes do grupo diabetes gestacional e para 52,8\% das classificadas no diabetes clínico, não havendo diferença entre os grupos. Resultados similares foram observados para a faixa de $\mathrm{GP} \geq 16 \mathrm{~kg}$, respectivamente 42 e $52,8 \%$. Entretanto, maior proporção das portadoras de diabetes gestacional (36\%) teve incremento de peso inferior a $8 \mathrm{~kg}$, com diferença estatística em relação ao grupo diabetes clínico (17\%), conforme Tabela 1.

Tabela 1 - Características maternas e aspectos relacionados à terapêutica insulínica no diabetes gestacional e clínico.

\begin{tabular}{|c|c|c|c|}
\hline & $\begin{array}{c}\text { Diabetes } \\
\text { gestacional } \\
\text { n (\%) }\end{array}$ & $\begin{array}{c}\text { Diabetes } \\
\text { clínico } \\
\text { n (\%) }\end{array}$ & $\mathbf{p}$ \\
\hline \multicolumn{4}{|l|}{ Características maternas } \\
\hline Idade $>25$ anos & $44(88)$ & $41(77,4)$ & 0,122 \\
\hline Multiparidade & $46(92)$ & $36(67,9)$ & 0,002 \\
\hline IMC $>25 \mathrm{~kg} / \mathrm{m}^{2}$ & $44(88)$ & $31(58,5)$ & 0,001 \\
\hline Ganho de peso & & & 0,03 \\
\hline$<8 \mathrm{~kg}^{*}$ & $18(36)$ & $9(17)$ & \\
\hline de 8 a $16 \mathrm{~kg}$ & $21(42)$ & $28(52,8)$ & \\
\hline$>16 \mathrm{~kg}$ & $11(22)$ & $16(30,2)$ & \\
\hline \multicolumn{4}{|l|}{ Insulinoterapia } \\
\hline Idade gestacional do início & & & 0,000 \\
\hline$\leq 12$ semanas* & $2(4)$ & $25(47,2)$ & \\
\hline 13-26 semanas & $15(30)$ & $22(41,5)$ & \\
\hline$\geq 27$ semanas* & $33(66)$ & $6(11,3)$ & \\
\hline Duração do tratamento & & & 0,000 \\
\hline$\leq 10$ semanas* & $34(68)$ & $8(15,1)$ & \\
\hline $11-20$ semanas & $13(26)$ & $15(28,3)$ & \\
\hline$\geq 21$ semanas* & $3(6)$ & $30(56,6)$ & \\
\hline Número de aplicações/dia & & & 0,000 \\
\hline uma* & $21(42)$ & $3(5,7)$ & \\
\hline duas & $21(42)$ & $21(39,6)$ & \\
\hline três* & $8(16)$ & $29(54,7)$ & \\
\hline \multicolumn{4}{|l|}{ Evolução - incremento } \\
\hline fisiológico & $26(52)$ & $34(64,2)$ & 0,147 \\
\hline não-fisiológico & $24(48)$ & $19(35,8)$ & 0,147 \\
\hline
\end{tabular}

$I M C=$ índice de massa corporal pré-gravídico; ${ }^{*}(p=\leq 0,05)$, Teste de Fisher e Goodman.
O grupo com diabetes gestacional foi caracterizado por iniciar o tratamento mais tardiamente $(66 \%$ a partir da $27^{\mathrm{a}}$ semana) e usar insulina por menor tempo (68\% por tempo máximo de dez semanas), aplicada uma vez ao dia (42\% em dose única/dia). Estes resultados foram diferentes dos observados no grupo diabetes clínico, caracterizado por início precoce $(47,2 \%$ até 12 semanas), maior duração ( $56,6 \%$ por mais de 21 semanas) e maior número de aplicações diárias $(54,7 \%$ receberam três aplicações/dia). Apesar disso, o comportamento da evolução do incremento de insulina durante a gestação não diferenciou os grupos, sendo classificado como fisiológico na maioria das gestantes ( $52 \%$ no gestacional e 64,2\% no clínico) conforme Tabela 1.

A quantidade total de insulina administrada por dia no primeiro trimestre não diferenciou os grupos, sendo de 47,5 $\pm 31,8 \mathrm{UI} /$ dia no grupo com diabetes gestacional e de 56,1 $\pm 28,2 \mathrm{UI} /$ dia no diabetes clínico $(p=0,681)$. A partir deste momento, as doses de insulina foram significativamente diferenciadas, evidenciando maior quantidade nas portadoras de diabetes clínico. No segundo trimestre, as doses administradas foram de 40,3 $\pm 19,7 \mathrm{UI} /$ dia nas diabéticas gestacionais e de

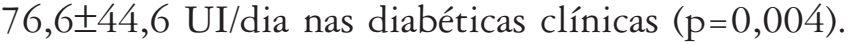
No terceiro trimestre, foram utilizadas $43,9 \pm 30,9$ e $92,0 \pm 50,1 \mathrm{UI} /$ dia de insulina $(\mathrm{p}<0,005)$, respectivamente nos grupos gestacional e clínico.

Relacionada ao peso real, administrou-se dose comparável de insulina nos dois grupos no primeiro trimestre de gestação, variando de $0,53 \pm 0,38 \mathrm{UI} / \mathrm{kg}$ peso no diabetes gestacional até $0,78 \pm 0,4 \mathrm{UI} / \mathrm{kg}$ peso no diabetes clínico, sem diferença significante $(\mathrm{p}=0,4)$. No segundo e terceiro trimestres da gestação, as pacientes com diabetes clínico receberam as maiores doses de insulina, respectivamente, $0,99 \pm 0,53 \mathrm{e}$ $1,12 \pm 0,56 \mathrm{UI} / \mathrm{kg}$ peso, diferenciando do grupo diabete gestacional, que recebeu em torno de $0,50 \mathrm{UI} / \mathrm{kg}$ peso (Tabela 2).

Maior proporção de diabéticas gestacionais recebeu doses menores que as preconizadas na literatura. De modo inverso, as diabéticas clínicas receberam doses maiores que as preconizadas, respectivamente, no segundo e terceiro trimestres da gestação. Estes resultados foram significativos (Tabela 2).

$\mathrm{O}$ incremento de insulina do primeiro para o terceiro trimestre da gestação foi de 0,12 e 0,43 UI/kg de peso real, no diabetes gestacional e no diabetes clínico, respectivamente (sem diferença $-\mathrm{p}=0,4$ ). Do segundo para o terceiro trimestre, o incremento foi de $0,37 \mathrm{UI} /$ $\mathrm{kg}$ nas diabéticas gestacionais e de 0,21 UI/kg entre as diabéticas clínicas, diferenciando estatisticamente os dois grupos avaliados $(\mathrm{p}=0,047)$. 
A MG materna mantida na gestação não mostrou diferença estatística entre os grupos. No primeiro trimestre, essa MG foi de 139,9 $\pm 20,0 \mathrm{mg} / \mathrm{dL}$ no diabetes gestacional e de $159,9 \pm 45,8 \mathrm{mg} / \mathrm{dL}$ no diabetes clínico

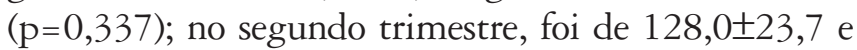
de $123,1 \pm 21,2 \mathrm{mg} / \mathrm{dL}(\mathrm{p}=0,358)$; e, no terceiro trimes-

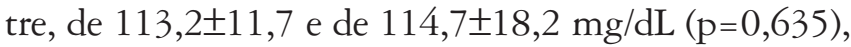
respectivamente, nas portadoras de diabetes gestacional e clínico.

Independentemente do tipo de diabete, a classificação da MG do terceiro trimestre da gestação evidenciou que o controle glicêmico foi ideal ( $\mathrm{MG} \leq 100 \mathrm{mg} / \mathrm{dL}$ ) ou adequado $(100<\mathrm{MG}<120 \mathrm{mg} / \mathrm{dL})$ na maioria das gestantes do estudo. Entretanto, o controle glicêmico foi inadequado ( $\mathrm{MG} \geq 20 \mathrm{mg} / \mathrm{dL}$ ) em maior proporção no diabetes clínico, diferente do observado no diabetes gestacional (Tabela 3).

A idade gestacional média no parto foi de $37,3 \pm 1,4$ semanas no diabetes gestacional e de $36,3 \pm 1$,9 semanas no diabetes clínico, com diferença significativa ( $\mathrm{p}=0,003)$ entre os grupos. Os resultados adversos perinatais mais comuns foram os recém-nascidos GIG e a hipoglicemia neonatal, respectivamente 30 e $26 \%$ no diabetes gestacional e 19,2 e 42,3\% no diabetes clínico, sem diferença estatística entre os grupos. A macrossomia foi o único resultado estatisticamente diferente entre os grupos $(\mathrm{p}=0,037)$, mais observada no diabetes gestacional (16 versus $3,8 \%$ ). SDR, policitemia, malformações e óbito fetal ocorreram em pequena proporção, apenas no diabetes clínico. A hiperbilirrubinemia foi diagnosticada somente no diabetes gestacional $(2 \%)$ e não ocorreu nenhum caso de morte neonatal. Na grande maioria dos recém-nascidos a alta hospitalar aconteceu nos primeiros sete dias e também não se relacionou ao tipo do diabetes materno (Tabela 3).

Tabela 2 - Dose de insulina (UI/ $\mathrm{kg}$ de peso atual) utilizada no Serviço e comparada com as doses propostas 9 a cada trimestre da gestação no diabetes gestacional e clínico.

\begin{tabular}{|c|c|c|c|}
\hline & $\begin{array}{c}\text { Diabetes } \\
\text { gestacional }\end{array}$ & $\begin{array}{c}\text { Diabetes } \\
\text { clínico }\end{array}$ & $\mathbf{p}$ \\
\hline Dose insulina (UI/kg peso) & $\mathrm{m} \pm \mathrm{Dp}$ & $\mathrm{m} \pm \mathrm{Dp}$ & \\
\hline $1^{\circ}$ trimestre & $0,53 \pm 0,38$ & $0,78 \pm 0,40$ & 0,400 \\
\hline $2^{\circ}$ trimestre & $0,50 \pm 0,30$ & $0,99 \pm 0,53$ & 0,001 \\
\hline $3^{\circ}$ trimestre & $0,49 \pm 0,39$ & $1,12 \pm 0,56$ & 0,000 \\
\hline Comparação das doses (UI/kg peso) & n $(\%)$ & n $(\%)$ & \\
\hline $1^{\circ}$ trimestre & & & 0,683 \\
\hline$<0,7$ & $1(50)$ & $11(42,3)$ & \\
\hline$\geq 0,7$ & $1(50)$ & $15(57,7)$ & \\
\hline $2^{\circ}$ trimestre & & & 0,001 \\
\hline$<0,8$ & $13(86,7)$ & $17(38,6)$ & \\
\hline$\geq 0,8$ & $2(13,3)$ & $27(61,4)$ & \\
\hline $3^{\circ}$ trimestre & & & 0,000 \\
\hline$<0,9$ & $45(90)$ & $25(48,1)$ & \\
\hline$\geq 0,9$ & $5(10)$ & $27(51,9)$ & \\
\hline
\end{tabular}

$M \pm D P=$ média \pm desvio padrão.

\section{Discussão}

Os resultados deste trabalho reforçam a hipótese de que o diabetes clínico difere do gestacional nos aspectos relacionados à insulinoterapia e, conseqüentemente, à qualidade do controle glicêmico materno e aos resultados perinatais. $\mathrm{O}$ grupo com diabetes clínico recebeu insulina mais precocemente, por período mais prolongado e em maior número de aplicações diárias. Considerando o peso real da gestante, este grupo também recebeu maior dose de insulina (UI/kg) que o grupo diabetes gestacional e que a recomendada na literatura, a partir do segundo trimestre de gestação. De modo inverso, o incremento do uso de insulina, do segundo para o terceiro trimestre da gestação, foi mais acentuado no grupo diabetes gestacional e maior proporção de gestantes do grupo diabetes clínico apresentou MG inadequada ( $\geq 120 \mathrm{mg} / \mathrm{dL}$ ) no final da gestação. Apesar disso, o único resultado perinatal adverso com freqüência diferente entre os grupos foi a ocorrência de macrossomia, mais freqüente no grupo diabetes gestacional.

Alguns trabalhos não confirmaram diferença na idade gestacional do início da insulinoterapia entre gestantes portadoras de diabetes gestacional e clínico ${ }^{12}$. Neste estudo, $66 \%$ das diabéticas gestacionais foram identificadas e tratadas a partir da $27^{\mathrm{a}}$ semana de gestação e $47,2 \%$ das diabéticas clínicas iniciaram o pré-natal até a $12^{\mathrm{a}}$

Tabela 3 - Controle glicêmico materno no terceiro trimestre da gestação e resultados perinatais no diabetes gestacional e clínico.

\begin{tabular}{|c|c|c|c|}
\hline & $\begin{array}{c}\text { Diabetes } \\
\text { gestacional } \\
\text { n (\%) }\end{array}$ & $\begin{array}{c}\text { Diabetes } \\
\text { clínico } \\
\text { n }(\%)\end{array}$ & $\mathbf{p}$ \\
\hline \multicolumn{4}{|l|}{ Controle glicêmico } \\
\hline$M G$ no 30 trimestre $(\mathrm{mg} / \mathrm{dL})$ & & & 0,042 \\
\hline$\leq 100$ & $5(10)$ & $10(19,6)$ & \\
\hline $100-120$ & $33(66)$ & $21(41,2)$ & \\
\hline$\geq 120^{*}$ & $12(24)$ & $20(39,2)$ & \\
\hline \multicolumn{4}{|l|}{ Resultados perinatais } \\
\hline GIG (peso>P90) & $15(30)$ & $10(19,2)$ & 0,151 \\
\hline Macrossomia (peso $\geq 4 \mathrm{~kg}$ ) & $8(16)$ & $2(3,8)$ & 0,037 \\
\hline Hiperbilirrubinemia (Bl>12 mg/dL) & $1(2)$ & $0(0)$ & 0,490 \\
\hline Hipoglicemia (glicemia $\leq 45 \mathrm{mg} / \mathrm{dL}$ & $13(26)$ & $22(42,3)$ & 0,630 \\
\hline Policitemia (HT>60\%) & $0(0)$ & $1(1,9)$ & 0,510 \\
\hline SDR & $0(0)$ & $2(3,8)$ & 0,257 \\
\hline Malformações & $0(0)$ & $2(3,8)$ & 0,257 \\
\hline Óbito fetal & $0(0)$ & $1(1,9)$ & 0,515 \\
\hline Óbito neonatal & - & - & - \\
\hline \multicolumn{4}{|l|}{ Tempo de internação } \\
\hline até 3 dias & $25(50)$ & $17(32,7)$ & 0,1238 \\
\hline 4 a 7 dias & $23(46)$ & $29(55,8)$ & \\
\hline$>7$ dias & $2(4)$ & $6(11,5)$ & \\
\hline
\end{tabular}

* $(p<0,05)$, Teste de Fisher e Goodman; $M G=$ média glicêmica materna; $G I G=$ recém-nascido grande para idade gestacional; BI=bilirrubina indireta; $\mathrm{HT}=$ hematócrito; SDR=Síndrome do desconforto respiratório. 
semana de gestação. A época de instituição de insulina pode ser influenciada pelo ingresso tardio no pré-natal, fato comum nas gestações associadas ao diabetes clínico e, também, pelo diagnóstico ou tratamento em fases mais adiantadas no diabetes gestacional. Outros relatos evidenciam que, apesar do diagnóstico prévio de diabetes, cerca da metade das gestantes inicia o pré-natal antes do segundo trimestre da gestação ${ }^{14}$. Esse fato também foi observado anteriormente entre nós e motivou esforços para a conscientização das puérperas diabéticas ${ }^{5}$.

Nos dois últimos trimestres da gestação, o grupo diabetes clínico recebeu maior quantidade de insulina, avaliada pela dose total diária (UI/dia) ou relacionada ao peso real da gestante (UI/kg). Estes dados ratificaram resultados anteriores 5 . Entretanto, o incremento de insulina (UI/ $\mathrm{kg}$ ) no final da gestação foi mais elevado no grupo diabetes gestacional.

Entre as pacientes com diabetes clínico, acentua-se a necessidade de insulina a partir da $25^{\mathrm{a}}$ semana, relacionada à resistência insulínica e à dificuldade no controle glicêmico materno ${ }^{15}$. A resistência à ação da insulina é resultado da produção hormonal placentária, incrementada a partir da segunda metade da gestação normal ou daquela complicada pelo diabetes. Tal efeito é mais acentuado no diabetes clínico tipo 2 e no diabetes gestacional, sendo também influenciada por características próprias da gestante, como sobrepeso e obesidade. A interação resistência insulínica, sobrepeso/ obesidade e gravidez constitui risco para o desenvolvimento de diabetes gestacional, favorecendo não só o diagnóstico da doença como as crescentes necessidades de insulina na segunda metade da gestação ${ }^{16}$. Assim, a proporção de $88 \%$ de diabéticas gestacionais com IMC pré-gravídico mínimo de $25 \mathrm{~kg} / \mathrm{m}^{2}$ deve ter influenciado o maior incremento de insulina (UI/kg) no diabetes gestacional. Esta proporção foi superior aos $58,5 \%$ de sobrepeso/obesidade observado no grupo diabetes clínico, inferindo resistência periférica mais acentuada e explicando a maior necessidade de insulina neste grupo de gestantes.

A análise do protocolo de insulinoterapia adotado demonstrou que menor quantidade de insulina por quilo de peso (UI/kg) foi administrada às diabéticas gestacionais e que as diabéticas clínicas receberam maior quantidade deste hormônio no final da gestação. Esses resultados foram comparados aos apresentados na literatura ${ }^{9}$. Na falta de ensaios clínicos e de estudos semelhantes na literatura, a qualidade do controle glicêmico materno e os resultados perinatais foram comparados para avaliar a necessidade de mudança no protocolo de insulina do serviço.

A menor quantidade de UI/kg de insulina, em média $0,5 \mathrm{UI} / \mathrm{kg}$, em qualquer trimestre, administrada às diabéticas gestacionais, relacionou-se ao controle glicêmico de melhor qualidade. Cerca de $80 \%$ delas manteve níveis de $M G$ ideais ou adequados (até $120 \mathrm{mg} / \mathrm{dL}$ ) a partir do segundo trimestre da gestação. No grupo diabetes clínico, que recebeu maior quantidade de insulina nos três trimestres, respectivamente $0,8,1,0$ e 1,1 UI/kg, a $\mathrm{MG}<120 \mathrm{mg} / \mathrm{dL}$ ocorreu em menor proporção de gestantes $(60 \%)$, o que poderia resultar em pior desfecho perinatal. Entretanto, tais resultados não diferenciaram os tipos de diabetes, exceto pela ocorrência de maior proporção de macrossomia no diabetes gestacional (16 versus 3,8\%). Mesmo assim, estes índices foram inferiores aos observados na literatura, que variaram de 21 até 56,1\% $14,17,18$.

Na prática, a obtenção do controle glicêmico ideal é dificultada ${ }^{14}$ e depende não só da insulinoterapia, mas de sua associação com dieta, exercício físico e, principalmente, adesão da gestante ${ }^{19}$. Nas gestações complicadas pelo diabetes, a macrossomia/crescimento fetal exagerado tem origem multifatorial, relacionada não só aos níveis glicêmicos maternos elevados no final da gestação, como a outros fatores de risco. A idade e a multiparidade, o IMC pré-gravídico $\geq 25 \mathrm{~kg} / \mathrm{m}^{2}$, o GP $>16 \mathrm{~kg}$ e, em especial, o antecedente de macrossomia representaram risco elevado para este desfecho perinatal adverso ${ }^{4,8,12,13,20}$. Neste estudo, o diferencial de $92 \%$ (versus $67,9 \%$ ) de multíparas e de $88 \%$ (versus $58,5 \%$ ) de gestantes com sobrepeso/obesas no diabetes gestacional deve ter contribuído para o alto índice de macrossomia. Vale ressaltar que este percentual só não foi maior porque $36 \%$ das mães deste grupo tiveram ganho de peso inferior $\mathrm{a}<8 \mathrm{~kg}$, provavelmente relacionado à dieta mais restritiva associada à prática regular de atividade física.

Nenhum dos demais resultados perinatais analisados diferenciou o diabetes clínico do gestacional, com percentuais sempre menores que os da literatura. A ocorrência de recém-nascidos GIG já foi mais elevada no serviço, atingindo cifras de 50 e 38\% estudos demonstraram que a hiperbilirrubinemia é responsável por cerca de $20 \%$ da morbidade neonatal, a hipoglicemia atinge índices de $64 \%$ e a policitemia acomete até $30 \%$ dos filhos de mães portadoras de diabetes. A SDR é diagnosticada entre 5 e $16 \%$ e o percentual de malformações corresponde a $12,3 \%$ nas gestações associadas ao diabetes clínico. O óbito fetal pode alcançar cifras de $14,2 \%$ no diabetes tipo 1 e de $10,3 \%$ no diabetes tipo 2 , e a mortalidade neonatal acomete de 2,8 a 6,2\% dos recém-nascidos destas gestantes $^{12,14,15,21,22}$. Independentemente do tipo de diabetes, os percentuais observados neste estudo ficaram muito aquém dos índices da literatura, indicando adequação do protocolo de tratamento.

Apesar de os grupos serem semelhantes entre si, uma análise mais crítica dos resultados chama atenção para os 
índices de $30 \%$ de recém-nascidos GIG no grupo diabetes gestacional e de 26 e 42,3\% de hipoglicemia neonatal, respectivamente, nos grupos diabetes gestacional e clínico. As características das gestantes multíparas e com sobrepeso/obesas, diferenciadas no diabetes gestacional, certamente favoreceram o crescimento fetal exagerado e, em especial, a grande proporção de GIG e macrossômicos neste grupo ${ }^{8,12,15}$. Nos dois grupos, a hipoglicemia neonatal deve ter sido mais freqüente pela diferenciação no critério diagnóstico. A literatura preconiza a confirmação do resultado, ou seja, exige pelo menos duas dosagens de glicemia neste limite ( $45 \mathrm{mg} / \mathrm{dL})^{4}$ e, em nosso serviço, um único valor neste limite foi considerado suficiente para confirmar hipoglicemia neonatal.
Do ponto de vista prático, alguns ajustes na associação dieta/exercício/insulina poderão melhorar a qualidade do controle glicêmico materno e as conseqüentes repercussões perinatais. Os resultados apontaram para a necessidade de aumento na quantidade de insulina no diabetes gestacional e individualização nos três trimestres da gestação. Apesar da maior quantidade de insulina administrada no diabetes clínico, o controle glicêmico deve ser intensificado. Assim, a restrição mais rigorosa da dieta e o estímulo ao exercício físico, sobretudo no diabetes tipo 2 , deverão melhorar o percentual de média glicêmica materna ideal/adequada e minimizar a morbidade perinatal nestas gestações complicadas pelo diabetes.

\section{Referências}

1. American Diabetes Association. Diagnosis and classification of diabetes mellitus. Diabetes Care. 2006;29 Suppl 1:S43-8.

2. American Diabetes Association. Gestational diabetes mellitus. Diabetes Care. 2004;27 Suppl 1:S88-90.

3. Gabbe SG, Graves CR. Management of diabetes mellitus complicating pregnancy. Obstet Gynecol. 2003;102(4):857-68.

4. Langer $O$, Yogev Y, Most $O$, Xenakis EM. Gestational diabetes: the consequences of not treating. Am J Obstet Gynecol. 2005; 192(4):989-97.

5. Rudge MVC, Calderon IMP, Ramos MD, Maestá I, Rugolo LMS, Peraçoli JC. Perspectiva perinatal decorrente do rígido controle pré-natal em gestações complicadas pelo diabete. Rev Bras Ginecol Obstet. 1995;17(1):26-32.

6. Rudge MV, Calderon IM, Ramos MD, Abbade JF, Rugolo LM. Perinatal outcome of pregnancies complicated by diabetes and by maternal daily hyperglycemia not related to diabetes. A retrospective 10-year analysis. Gynecol Obstet Invest. 2000;50(2):108-12.

7. Landon MB, Catalano PM, Gabbe SG. Diabetes mellitus. In: Gabbe SG, Niebyl JR, Simpson JL, editors. Obstetrics: normal and problem pregnancies. Philadelphia: Churchil Livingstone; 2002. p. 1009-100.

8. Kerche LTRL, Abbade JF, Costa RAA, Rudge MVC, Calderon IMP. Fatores de risco para macrossomia fetal em gestações complicadas por diabete ou por hiperglicemia diária. Rev Bras Ginecol Obstet. 2005;27(10):580-7.

9. de Veciana M, Major CA, Morgan MA, Asrat T, Toohey JS, Lien JM, et al. Postprandial versus preprandial blood glucose monitoring in women with gestational diabetes mellitus requiring insulin therapy. N Engl J Med. 1995;333(19):1237-41.

10. Homko CJ, Khandelwal M. Glucose monitoring and insulin therapy pregnancy. Obstet Gynecol Clin North Am. 1996;23(1):47-74.

11. Ministério da Saúde. Assistência pré-natal: manual técnico. 3a ed. Brasília: Ministério da Saúde; 2000. p. 29-30.

12. Montenegro Júnior RM, Paccola GMFG, Faria CM, Sales APM, Montenegro APDR, Jorge SM, et al. Evolução materno- fetal de gestantes diabéticas seguidas no HC-FMRP-USP no período de 1992-1999. Arq Bras Endocrinol Metab. $2001 ; 45(5): 467-74$.

13. Langer O, Conway DL, Berkus MD, Xenakis EM, Gonzales O. A comparison of glyburide and insulin in women with gestational diabetes mellitus. N Engl J Med. 2000;343(16):1 134-8.

14. Corrêa FHS, Gomes MB. Acompanhamento ambulatorial de gestantes com diabetes mellitus no Hospital Universitário Pedro Ernesto-UERJ. Arq Bras Endocrinol Metab. 2004;48(4):499-504.

15. Carvalho MM, Mendonça VA, Alencar Júnior CA, Augusto APA, Nogueira MB. Estudo comparativo dos resultados maternos e perinatais entre pacientes com diabetes pré-gestacional tipo I e tipo II. Rev Bras Ginecol Obstet. 2000;22(5):257-63.

16. Calderon IMP, Rudge MVC. Insulinoterapia no ciclo gravídico-puerperal. Benefícios e controvérsias. Femina. 1997;25(6):533-7.

17. Roland JM, Murphy HR, Ball V, Northcote-Wright J, Temple RC. The pregnancies of women with type 2 diabetes: poor outcomes but opportunities for improvement. Diabet Med. 2005;22(12):1774-7

18. Silva JC, Taborda W, Becker F, Aquim G, Viese J, Bertini AM. Resultados preliminares do uso de anti-hiperglicemiantes orais no diabete melito gestacional. Rev Bras Ginecol Obstet. $2005 ; 27(8): 461-6$.

19. Rudge MVC, Calderon IMP, Kerche LTRL. Diabete na gravidez. In: Ramos JG, Urbanetz AA, editores. Programa de Atualização em Ginecologia e Obstetrícia (Proagro): sistema de educação médica continuada a distância: módulo 2/ciclo 2. Porto Alegre: Artmed; 2004. p. 9-47.

20. Guerra G, Costelo A, Santos LC. Case management survey of diabetes in pregnancy. Rev Cienc Med. 2004;13(3):193-204.

21. Maganha CA, Bernardini MA, Vanni DGBS, Nomura RMY, Zugaib $M$. Repercussões do diabetes no feto e recém-nascido. Rev Ginecol Obstet. 2002;13(3):158-62.

22. Evers IM, de Valk HW, Visser GH. Risk of complications of pregnancy in women with type 1 diabetes: nationwide prospective study in the Netherlands. BM. 2004;328(7445):915-9. 